Case Report

Open Access

\title{
Haptic Reposition with Laser Posterior Capsulotomy in Eyes with Capsular Contraction without Anterior Capsular Phimosis
}

\author{
Ayse Gul Kocak Altintas ${ }^{1 \star}$ and Cagri Ilhan ${ }^{2}$ \\ ${ }^{1}$ Associated Professor, University of Health Sciences, Ankara Ulucanlar Eye Education and Research Hospital, Ankara, Turkey \\ ${ }^{2} M D$, Hatay State Hospital, Hatay, Turkey
}

\section{Article Info \\ *Corresponding author: \\ Ayse Gul Kocak Altintas \\ Associated Professor \\ University of Health Sciences \\ Ankara Ulucanlar Eye Education and \\ Research Hospital \\ Ulucanlar Cad. No: 5906230 \\ Altindag, Ankara \\ Turkey \\ Tel: 90-312-3126261 \\ Fax: 90-312-3124827 \\ E-mail: aysegulkaltintas@hotmail.com}

\section{Received: June 7, 2018}

Accepted: July 23, 2018

Published: July 27, 2018

Citation: Altintas AGK, Ilhan C. Haptic Reposition with Laser Posterior Capsulotomy in Eyes with Capsular Contraction without Anterior Capsular Phimosis. Madridge J Ophthalmol. 2018; 3(1): 36-38.

doi: 10.18689/mjop-1000111

Copyright: (c) 2018 The Author(s). This work is licensed under a Creative Commons Attribution 4.0 International License, which permits unrestricted use, distribution, and reproduction in any medium, provided the original work is properly cited.

Published by Madridge Publishers

\begin{abstract}
Capsular contraction (CC) is one of the important complication of the cataract surgery. In rare cases, CC may cause intraocular lens (IOL) decentration, dislocation or dysmorphology. 64 year-old patient underwent cataract surgery nearly 8 months ago, presented with decreased vision due to $\mathrm{CC}$ and full flexion of the inferior haptic onto the posterior optic surface of the single piece hydrophylic acrylic IOL. We treated this unusual complication with neodymium:yttrium-aliminum-garnet (Nd:YAG) laser posterior capsulotomy without any surgical manipulation. Folded inferior haptic of IOL was immediately repositioned after the release of posterior capsular tension. In conclusion, Nd:YAG laser posterior capsulotomy is an effective and minimally invasive treatment method in cases with full haptic flexion onto the posterior optical surface of the IOL.
\end{abstract}

Keywords: Haptic Reposition; Laser Posterior Capsulotomy; Capsular Contraction (CC); Anterior Capsular Phimosis.

\section{Introduction}

Posterior capsular opacification (PCO) is the most common complication of cataract surgery that occur due to lens epithelial cell (LEC) proliferation and migration to the posterior capsular surface [1]. Anterior LEC metaplasia, proliferation leads anterior capsular opacification (ACO) similar to PCO, in addition LEC myofibroblastic transformation to actine positive smooth muscle cause fibrotic anterior capsular contraction (CC) [2]. An imbalance between centrifugal and centripedal forces of the capsular bag due to zonular weakness is the most important predisposing factor in the pathogenesis of CC [3]. CC may cause IOL folding, decentration, tilt or dislocation in extreme cases $[4,5]$. In this report, we presented a case with single piece hydrophilic acrylic IOL where of haptic folded on posterior surface of the optic due to CC without significant anterior capsular phimosis and we treated this unusual complication with neodymium:yttrium-aliminum-garnet (Nd:YAG) laser posterior capsulotomy without any surgical manipulation and laser of anterior capsule edges. As far as we know presenting case is the first report that showed the full flexion of the haptic onto the posterior surface of the optic due to CC without anterior capsular phimosis and benefits of laser posterior capsulotomy without anterior capsule manipulation. 


\section{Case Report}

Sixty-four year-old, Caucasian female patient presented with complaint of decreased vision in her left eye after cataract surgery. Auto kerato-refractometer device cannot indicate any measurement for the left eye and shows low myopic astigmatic refraction for the phakic right eye. Intraocular pressure was measured as $16 \mathrm{mmHg}$ bilaterally by pneumatic tonometer. In the examination of left eye, uncorrected visual acuity was 20/400, ocular surface and anterior chamber were normal but PCO and folding of the IOL haptic were seen in pupillary aperture. Circular anterior capsular opacification, anterior CC, minimal PCO and full flexion of the inferior haptic onto the posterior optic surface were seen after pupillary dilation of the left eye (Figure 1). There were posterior vitreous detachment and retinal pigment epithelial alterations in posterior segment. In the examination of right eye, 20/25 best corrected visual acuity, nuclear sclerotic cataract and similar fundoscopic findings were seen.

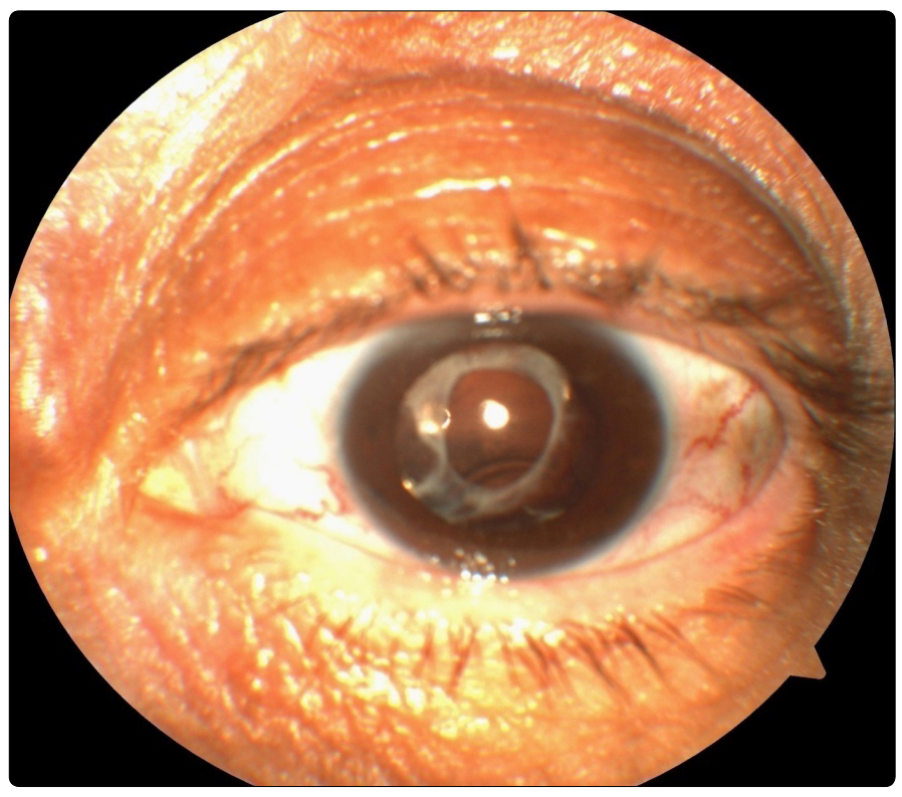

Figure 1. Circular anterior capsular opacification, anterior CC, minimal PCO and full flexion of the inferior haptic onto the posterior optic surface are seen

According to her medical reports, she had undergone uneventful phacoemulsification and $21 \mathrm{D}$ single piece hydrophilic acrylic IOL implantation 8 months ago and visual acuity of the patient was decreased gradually for last 2 months. There is no history of ocular, cranial or systemic trauma after surgery and the patient had not any ocular and systemic diseases except type II diabetes mellitus and nonproliferative diabetic retinopathy. She mentioned regularly using of long acting insulin for 4 years. Nevertheless blood sugar of the patient can be high for the comment of the patient.

For the excessive anterior $\mathrm{CC}$ and posterior capsule opacification (PCO), we performed Nd:YAG laser capsulotomy as our first preference by comparison with surgical manipulation. After the informed consent of the patient, standard preoperative laser posterior capsulotomy procedures including pupillary dilation, topical anesthesia were applied.
Contracted area in upper temporal quadrant of posterior capsule were opened with 6 shoots of $10 \mathrm{~J} \mathrm{Nd:YAG} \mathrm{laser}$ application and folded inferior haptic of IOL was immediately repositioned after the release of posterior capsular tension (Figure 2). We did not perform further laser application when considering clearance of the rest of posterior capsule and being of the patient in relatively high risk period for cystoid macular edema, then treatment was finalized. Her best corrected visual acuity improved from 20/400 to 20/40 immediately after the laser treatment. $\mathrm{OL}$ remained the same without any folding at the last visit of 4 month.

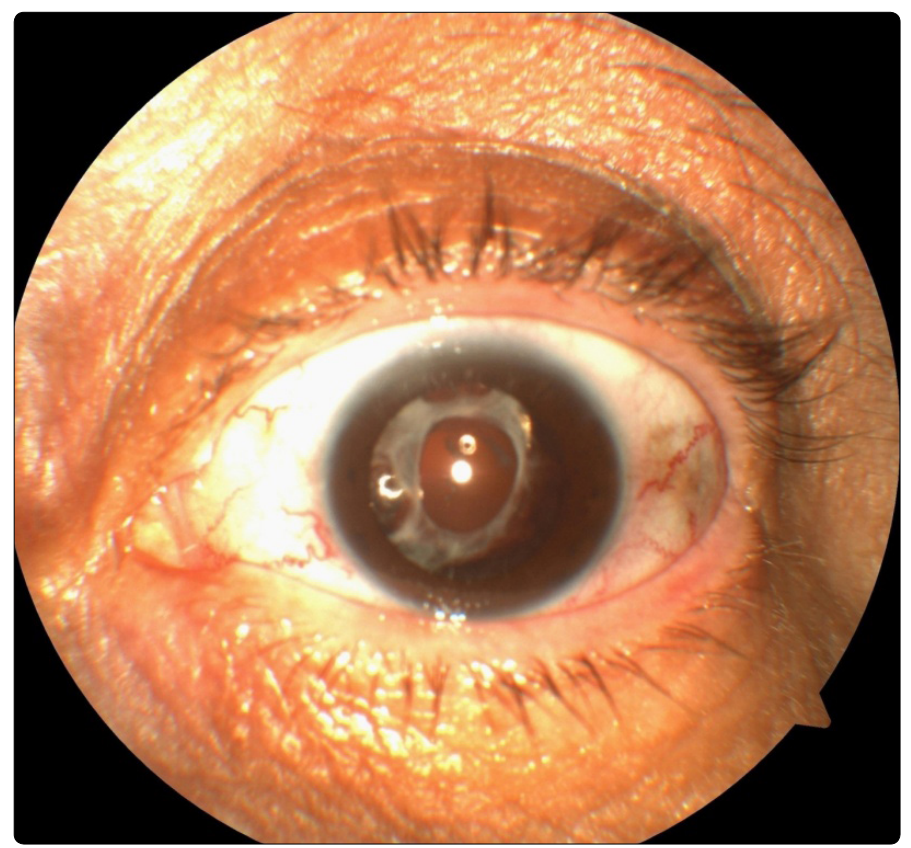

Figure 2. Repositioned inferior haptic of IOL is seen after the release of posterior capsular tension

\section{Discussion}

Michael et al. [6] stated 4 cases of capsule contraction causes full flexion of the haptics onto the anterior surface of the hydrophilic acrylic IOLs optics and the authors advised surgical procedures for patients in their series. Altintas et al. [7] reported a cases with significant folding of both haptic and optic edge over the central part of IOL and they performed surgical capsulectomy rather than Nd:YAG laser capsulotomy. It was reported that a patient with Behçet's disease, capsulotomy opening was occuded by fibrotic membrane several months after Nd:YAG laser capsulotomy and patient had to undergone surgical capsulectomy [8]. In our case, full flexed haptic contacts the posterior surface of the optic without prominent shrinkage of anterior capsular opening. We firstly consider Nd:YAG laser posterior capsulotomy for the purpose of both to reduce posterior capsular tension and treat PCO. After the opening of posterior capsule, the tension reduced and full flexed haptic immediately repositioned, then we did not need further manipulation. Deokule at al. [9] were observed severe CC syndrome and performed Nd:YAG laser capsulotomy without any surgical treatment similar to our case.In this regard, our patient is more lucky by comparison with cases of underwent surgery because she was prevented 
to potential risks of surgical manipulations including ciliary body detachment, hypotony and supraciliary effusions $[6,7]$.

Zonular weakness due to pseudoexfoliation syndrome, myotonic muscular dystrophy, retinitis pigmentosa, advanced age, chronic intraocular inflammation, and trauma (including surgical trauma) plays a key role in the pathogenesis of CC [3,8-12]. In series of Michael et al. [6] all subjects had a risk factor affects the zonular stability such as uveitis, advanced age and retinitis pigmentosa, but our case is the absence of any systemic and ocular risk factors rather than diabetes that have the potential of the influence the zonular stability [8]. Nevertheless, previous ocular surgery such as capsulorhexis size, IOL design and materials can be considered as an important predisposing factor to facilitate the formation of anterior CC.

Actually, anterior CC occurs more frequently after silicone or hydrogel IOL implantation due to a weaker adhesion between optical surface and lens capsule [10-13]. In contrast, mainly hydrophobic acrylic material adheres more firmly and these adhesions restrict LEC proliferation and migration $[11,14]$. Nevertheless, anterior CC may cause excessive contraction of total lens equator size and full flexion of haptic of acrylic IOL. The interesting part of our case is posterior folding of acrylic IOL haptic onto the posterior optic surface between potential space of optic and posterior capsular bag. Even hydrophilic acrylic IOL adhesions to the posterior lens surface was not as strong as hydrophobic acrylic one, posterior folding of haptic in our case indicated that the adhesion was weaker than expected for any type of IOL, as far as we know which was not reported before us.

Another interesting observation from our patient that capsulotomy opening was neither small nor constricted,in contrast, only PCO was observed combined with total shrinkage of lens equator diameter that cause haptic bending.

As far as we know, ours is the first case had posterior bending of the haptic onto the posterior optic surfaceof the hydrophilic acrylic IOL without prominent anterior $\mathrm{CC}$, that treated with Nd:YAG laser posterior capsulotomy which is an effective and minimally invasive treatment method.

\section{References}

1. Awasthi N, Guo S, Wagner BJ. Posterior capsular opacification; a problem reduced but not yet eradicated. Arch Ophthalmol. 2009; 127(4): 555-562. doi: 10.1001/archophthalmol.2009.3

2. Hayashi $\mathrm{K}$, Hayashi $\mathrm{H}$. Intraocular lens factors that may affect anterior capsule contraction. Ophthalmology. 2005; 112(2): 286-292. do: 10.1016/j. ophtha.2004.11.013

3. Kramer GD, Werner L, Neuhann T, Tetz M, Mamalis N. Anterior haptic flexing and in-the-bag subluxation of an accommodating intraocular lens due to excessive capsular bag contraction. J Cataract Refract Surg. 2015; 41(9): 2010-2013. doi: 10.1016/j.jcrs.2015.08.009

4. Ohmi S. Decentration associated with asymmetric capsular shrinkage and intraocular lens size. J Cataract Refract Surg. 1993; 19(5): 640-643.

5. Kim MH, Chung TY, Chung ES. Long-term efficacy and rotational stability of AcrySof toric intraocular lens implantation in cataract surgery. Korean J Ophthalmol. 2010; 24(4): 207-212. doi: 10.3341/kjo.2010.24.4.207

6. Michael K, O'Colmain U, Vallance JH, Cormack TG. Capsule contraction syndrome with haptic deformation and flexion. J Cataract Refract Surg. 2010; 36(4): 686-689. doi: 10.1016/j.jcrs.2009.09.042

7. Altintas AGK, Dal D, Simsek S. Significant intraocular lens folding due to severe capsular contraction. Jpn J Ophthalmol. 2008; 52(2): 134-136. doi: 10.1007/s10384-007-0500-z

8. Alintas AGK, Dal D. Capsule contraction syndrome in Behcet's disease. Int J Ophthalmol. 2010; 3(4): 358-360. doi: 10.3980/j.issn.2222-3959.2010.04.20

9. Deokule SP, Mukherjee SS, Chew CKS. Neodymium: YAG laser anterior capsulotomy for capsular contraction syndrome. Ophthalmic Surg Lasers Imaging Retina. 2006; 37(2): 99-105. doi: 10.3928/1542-8877-20060301-04

10. Salzmann J, Khaw PT, Laidlaw A. Choroidal effusions and hypotony caused by severe anterior lens capsule contraction after cataract surgery. Am J Ophthalmol. 2000; 129(2): 253-254. doi: 10.1016/S0002-9394(99)00319-0

11. Tognetto D, Toto L, Sanguinetti G, Cecchini P, Vattovani O, Filacorda S, et al. Lens epithelial cell reaction after implantation of different intraocular lens materials: two-year results of a randomized prospective trial. Ophthalmology. 2003; 110(10): 1935-1941. doi: 10.1016/S0161-6420(03)00736-X

12. Kato $S$, Suzuki $T$, Hayashi $Y$, Numaga J, Hattori $T$, Yuguchi $T$, et al. Risk factors for contraction of the anterior capsule opening after cataract surgery. J Cataract Refract Surg. 2002; 28(1): 109-112.

13. Cochener B, Jacq PL, Colin J. Capsule contraction after continuous curvilinear capsulorhexis: poly(methylmethacrylate) versus silicone intraocular lenses. J Cataract Refract Surg. 1999; 25(10): 1362-1369. doi: 10.1016/S08863350(99)00227-8

14. Kim SY, Yang JW, Lee YC, Kim SY. Effect of haptic material and number of intraocular lens on anterior capsule contraction after cataract surgery. Korean J Ophthalmol. 2013; 27(1): 7-11. doi: 10.3341/kjo.2013.27.1.7 